\title{
Echo-Planar Imaging
}

National Cancer Institute

\section{Source}

National Cancer Institute. Echo-Planar Imaging. NCI Thesaurus. Code C17558.

A magnetic resonance imaging (MRI)-based technique in which multiple lines of imaging data are acquired after each radiofrequency excitation. This allows images to be obtained in 20-100 ms rather than over several minutes, resulting in increased temporal resolution compared to other MRI methods. 